\title{
Research on a High Temperature Multi-Mode Cavity Resonator Designing for Deoxidizing Ilmenite
}

\author{
Chen Hua ${ }^{1, a}$, Guo Shenghui ${ }^{2, b}$, Zhou Junwen ${ }^{2, ~ c}$, Peng Jinhui ${ }^{2, d}$ \\ ${ }^{1}$ Faculty of Science, Kunming University of Science and Technology, Kunming, 650500, China \\ ${ }^{2}$ Faculty of Metallurgy and Energy Engineering, Kunming University of Science and Technology, \\ Kunming, 650093, China \\ a email: cherrychen40600@163.com, bemail:shguo78@hotmail.com, 'email: 754400566 @qq.com, \\ demail: Jhpeng_ok@Yeah.net
}

Keywords: Multimode cavity; Equivalent impedance; HFSS software; Microwave heating

\begin{abstract}
The designing of microwave cavity reactor for microwave metallurgy is usually according to the nature and the dimensions of the product to be processed, the operating frequency, the number of modes and so on. In this paper, the main mode equivalent impedance matching is proposed to design the cavity resonator. The size of multimode cavity resonator is calculated by the approximate equivalent impedance method. On the sample of ilmenite for heating, the HFSS software validates the design of the multimode cavity resonator by method of equivalent impedance. The location of the transmission waveguide is also simulated by HFSS. The simulation results show that the theory result is reasonable. It can provide a reference for the designing of high temperature microwave metallurgy multimode cavity resonator.
\end{abstract}

\section{Introduction}

It is widely known that microwave (MW) heating can improve the efficiency and quality of a variety of applied thermal processes, like food processing, sterilization, pasteurization, drying of wood, vulcanization of rubber, waste processing and recycling, mineral processing, etc[1-3]. MW radiation causes internal heating of the material then lower temperatures and shorter times can be used compared to those applied at conventional heating. Nowadays, microwaves are typically used for food cooking. However, they are also utilized in industry to process absorbing materials like ceramics, evaporate water, and this technique has been extended to electrically conducting powder [4-5].

It is seldom on reported that building a mathematical model to make the transmission waveguides match with cavity in advance before designing. According to the transmission theory [6-8], it is known that a not good matching between transmission waveguides and cavity will result in microwave power reflected, heating nonuniform, even the magnetron destroyed. In this paper, the transmission waveguides matching with cavity is considered into design. The approximate equivalent impedance of cavity is derived. The dielectric properties of ilmenite were measured with an open-ended coaxial probe dielectric measurement system at a frequency of $2.45 \mathrm{GHz}$. On the load of ilmenite for heating, the HFSS software [9] validates the design is viable. The cavity is stable along with the temperature raised for heating ilmenite.

\section{Equivalent Impedance of transmission waveguide and rectangular cavity}

In this paper, the cavity resonator assumed has the size of $a=400 \mathrm{~mm}$ and $c=400 \mathrm{~mm}$ according to the experimental requirement, while the length of $b$ along the $y$ axis is uncertain.

The transmission waveguide selected in the microwave reactor has the size of $86.4 \mathrm{~mm} \times$ 43.2 $\mathrm{mm}$ which is shown in Fig. 1 . The operation frequency is $f=2.45 \mathrm{GHz}$, which means that the free space wavelength is $122 \mathrm{~mm}$. The microwave source via rectangular waveguide operates in the $T E_{10}$ mode, which is a wave that has no electric field component in the direction of propagation. $T E_{10}$ mode is the only propagation mode through the rectangular waveguide. The field components for 
$T E_{10}$ mode can be calculated from above equation (7) to (12). The multimode is excited when the microwave source comes into the cavity resonator because of the section area changed. According to the transmission theory, the reflection will be least if the impedance of the waveguide is equal to the equivalent impedance of cavity resonator. Thus the size of cavity resonator can be confirmed if the equivalent impedance of cavity resonator and waveguide can be calculated. In this paper, the walls of waveguide and cavity resonator are perfect conductors.

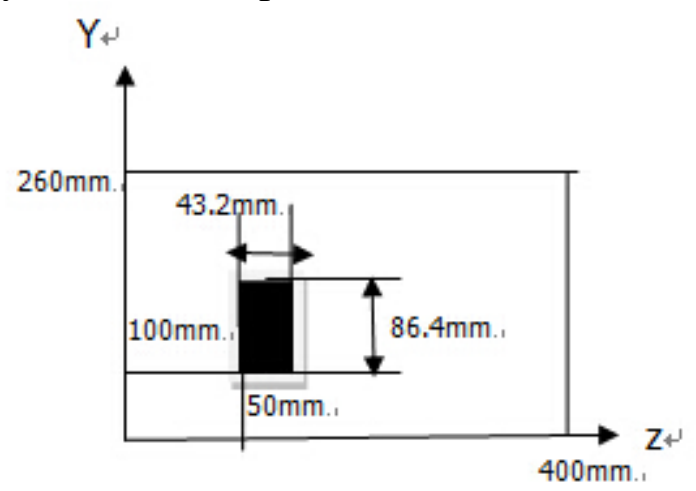

Fig.1. The location of transmission waveguides on rectangular cavity resonator

Assumed the $a^{\prime}=86.4 \mathrm{~mm}, \quad b^{\prime}=43.2 \mathrm{~mm}$, then the components for $T E_{10}$ mode

$$
\begin{aligned}
& H_{z}=H_{10} \cos \left(\frac{\pi}{a^{\prime}} x\right) e^{j(\omega t-\beta z)} \\
& H_{x}=H_{10} \frac{j \beta}{k_{c}^{2}}\left(\frac{\pi}{a^{\prime}}\right) \sin \left(\frac{\pi}{a^{\prime}} x\right) e^{j(\omega t-\beta z)} \\
& E_{y}=H_{10} \frac{-j \omega \mu}{k_{c}^{2}}\left(\frac{\pi}{a^{\prime}}\right) \sin \left(\frac{\pi}{a^{\prime}} x\right) e^{j(\omega t-\beta z)} \\
& E_{z}=0
\end{aligned}
$$

According to the circuit theory, the equivalent impedance of waveguide $Z_{e}$ can be calculated as

$$
Z_{e}=\frac{V_{e}^{+}}{I_{e}^{+}}
$$

where the $V_{e}^{+}$and $I_{e}^{+}$stand for the equivalent voltage and current of the waveguide.

Defined that the equivalent voltage is the line integral of center electric field from the top line to the bottom line

$$
\begin{aligned}
& V_{e}^{+}=\left.\int_{y=b^{\prime}}^{0} E_{y}\right|_{x=a^{\prime} / 2} d y=\int_{y=b^{\prime}}^{0}-\frac{j w \mu a^{\prime}}{\pi} H_{10} \sin \left(\frac{\pi}{2}\right) e^{-j \beta z} d y \\
& =\frac{j w \mu a^{\prime} b^{\prime}}{\pi} H_{10} e^{-j \beta z}
\end{aligned}
$$

Defined that the equivalent current is the sum of the current along the $a^{\prime}$.

$$
\begin{aligned}
& I_{e}^{+}=\int_{x=0}^{a^{\prime}} J_{z} d x=\int_{x=0}^{a^{\prime}} H_{x} d x=\int_{x=0}^{a^{\prime}}-\frac{j \beta a^{\prime}}{\pi} H_{10} \sin \left(\frac{\pi}{a^{\prime}} x\right) e^{-j \beta z} d x \\
& =\frac{j 2 \beta a^{\prime 2}}{\pi^{2}} H_{10} e^{-j \beta z}
\end{aligned}
$$

Let (6) and (7) in (5), we can get the waveguide equivalent impedance as:

$$
Z_{e}^{+}=\frac{V_{e}^{+}}{I_{e}^{+}}=\frac{\pi}{2} \frac{b^{\prime}}{a^{\prime}} \frac{\omega \mu}{\beta}=\frac{\pi}{2} \frac{b^{\prime}}{a^{\prime}} Z_{T E_{10}}=\frac{\pi}{2} \frac{b^{\prime}}{a^{\prime}} \frac{\eta}{\sqrt{1-\left(\lambda / 2 a^{\prime}\right)^{2}}}
$$

where $\eta$ is the wave impedance in free space. Here the equivalent impedance of cavity resonator is done with waveguide equivalent impedance of main mode, since the most of the power is included in $T E_{10}$. As a result, the equivalent impedance of cavity resonator can be written as: 


$$
Z \approx \frac{\pi}{2} \frac{b}{a} \frac{\eta}{\sqrt{1-(\lambda / 2 a)^{2}}}
$$

It can be think that the waveguide matches with cavity resonator when

$$
Z_{e}^{+}=Z
$$

Finally, we can get the size of $b$ by follow equation

$$
b=\frac{b^{\prime}}{a^{\prime}} \frac{a \sqrt{1-(\lambda / 2 a)^{2}}}{\sqrt{1-\left(\lambda / 2 a^{\prime}\right)^{2}}}
$$

Therefore we can see that the matching is decided to the length of the broad line and the ratio of the broad line to short line in section. So if we initially assumed that the cavity resonator has the size of $a=400 \mathrm{~mm}$ and $c=400 \mathrm{~mm}$, then we can calculate the length of $b \approx 235 \mathrm{~mm}$ by equation (11).

\section{The simulation results}

The Ansoft HFSS 11 software is utilized for simulation and optimized for cavity resonator. The theory value of $\mathrm{b} b \approx 235 \mathrm{~mm}$ by equation above, so the length of $b$ is set changing from $220 \mathrm{~mm}$ to $260 \mathrm{~mm}$ by HFSS, with the step $=5 \mathrm{~mm}$. The ilmenite is selected as sample for heating at $100^{\circ} \mathrm{C}$, which the electric permittivity is about 8.1594 and the dielectric loss is about 2.27484.

In Fig. 2(a), the VSWR changing with the length of b are shown. It can be seen that when the length of $b=255 \mathrm{~mm}$, the VSWR of waveguide becomes least, about 2.18, which mean the reflection is about 0.37 at this time and about $63 \%$ of power is fed into the cavity. The error between simulation and theory value is about $7 \%$. Then the length of $b$ is set $255 \mathrm{~mm}$, the location of waveguide is optimized. The initial distance between waveguide and $\mathrm{Y}$ axis is $100 \mathrm{~mm}$, while the initial distance between waveguide and $\mathrm{Z}$ axis is $50 \mathrm{~mm}$ shown in Fig.1. The distance between waveguide and $Z$ axis is first set changing from $50 \mathrm{~mm}$ to $120 \mathrm{~mm}$, with the step $=5 \mathrm{~mm}$.. It can be observed by Fig.2(b) that the VSWR become least when the distance between waveguide and $\mathrm{Z}$ axis is about $80 \mathrm{~mm}$. The VSWR is 1.53 at this time, which mean the reflection is about 0.2 at this time and about $80 \%$ of power is fed into the cavity.

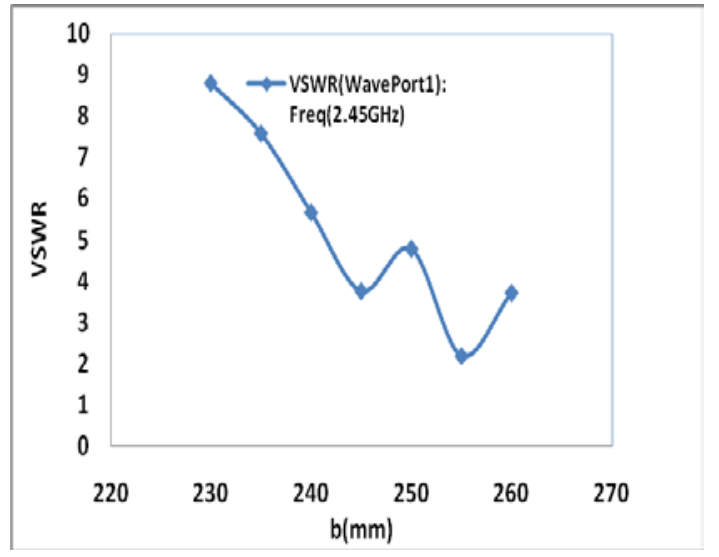

(a) The VSWR change with the length of $b$

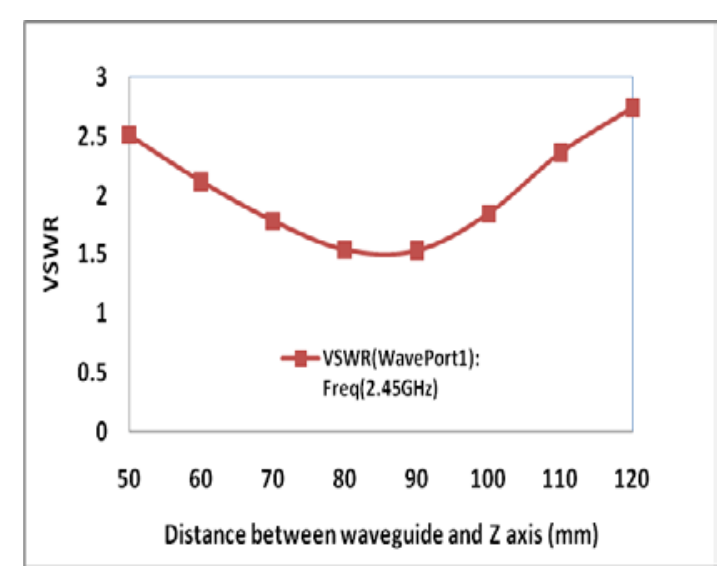

(b)Optimization of transmission waveguide Fig.2. Optimization of cavity and transmission waveguide

\section{Conclusion}

The design of cavity resonator based on the equivalent impedance is proposed in this work. The simulation and optimization results proved that the value calculated by the equivalent impedance is feasible and VSRW can satisfy with the engineering requirement. As the approximate equivalent impedance is utilized for cavity resonator and the highest-order modes are ignored which result in 
the simulation result is not consistent with the theory value. But the initial value set nearby the theory value short the designing time all the same. This work can provide a new method for the design of multi-mode cavity resonator and reduce the time of design procession.

\section{Acknowledgement}

The authors thank the support of the International S\&T Cooperation Program of China (No. 2012DFA70570), Yunnan Provincial Foundation Program (No. 2013FZ016), National Science Foundation of China (No. 51304097),National Science Foundation of China (No. 51264015).

\section{References}

[1] M. Oghbaei, O. Mirzaee, Microwave versus conventional sintering: A review of fundamentals, advantages and applications [J], Journal of Alloys and Compounds, vol.494, pp.175-189 , 2010.

[2] Chen H, Tang J, Liu F, “Coupled simulation of an electromagnetic heating process using the finite difference time domain_method,” J Microw Power Electromagn Energy, vol. 41, No.3, pp.50-68, 2007

[3] Y. Jian, X. Q. Yang, K. M. Huang "Numerical analysis of the influence of stir on water during microwave heating,” Progress In Electromagnetics Research C, Vol. 17, 105-119, 2010.

[4] R. Roy, D. Agrawal, J. Cheng and S. Gedevanishvili, "Full sintering of powdered-metal bodies in a microwave field” Nature(London), vol.399, pp.668-670, 1999.

[5] J. Cheng, R. Roy and D. Agrawal, "Experimental proof of major role of magnetic field losses in microwave heating of metal and metallic composites”, J. Mater. Sci. Lett., vol.20, pp.1561-1563, 2001

[6] J.R. Reitz, F. J. Milford and R.W.Christy, Foundations of Electromagnetic Theory. Addison Wesley, Reading, MA(1980)Addiso

[7] XIE Chu-fang, RAO Ke-jing, Electromagnetic Field and Electromagnetic Wave [M], High Education Press, 1999: 184-196.

[8] Chen H, Liu Chenghui, Zhou Junwen,etc., "Research on the multimode cavity designing for deoxidizing ilmenite based on waveguide equivalent impedance”, Materials Review, vol.28, pp.133-137, 2014.

[9] XIE Yong-jun, WANG Peng. Foundation and Application of Ansoft HFSS [M]. Xian: Xidian University Press, 2007: 30-96. 